\title{
Estudo comparativo entre tomografia computadorizada de alta resolução e radiografia de tórax no diagnóstico da silicose em casos incipientes"
}

\author{
Ana Paula Scalia Carneiro ${ }^{1}$, Arminda Lucia Siqueira ${ }^{2}$, Eduardo Algranti ${ }^{3}$, Cid SÉrgio Ferreira ${ }^{4}$, \\ Jorge Issamu KaVAKama ${ }^{5}$, Maria Luiza Bernardes ${ }^{6}$, Thaís Abreu DE CASTRO ${ }^{7}$, RenÉ MENDES ${ }^{8}$
}

\begin{abstract}
Introdução: A radiografia de tórax (RX) ainda é, no dias atuais, o principal método de diagnóstico da silicose, seguindo-se as normas da Organização Internacional do Trabalho (OIT). A interpretação radiológica de casos iniciais é difícil, podendo ocorrer divergências mesmo entre leitores experientes. Recentemente, tem sido considerada a possibilidade de utilização da tomografia computadorizada com técnica de alta resolução (TCAR) para avaliação de casos incipientes. Objetivo: Comparar os resultados da RX com a TCAR. Material e métodos: Foi avaliado um grupo inicial de 135 exmineiros, cujas radiografias foram examinadas por três leitores, no período de novembro de 1997 a dezembro de 1999. Indicou-se TCAR para 68 pacientes, cuja mediana de três leituras radiológicas foi menor ou igual a 1/0. As tomografias foram avaliadas por dois leitores e, em casos de divergência, houve participação de um terceiro leitor. As TCAR foram classificadas de acordo com a profusão de micronódulos em categorias de 0 a 3. Os resultados de TCAR e RX foram comparados através do teste de McNemar, coeficiente Kappa ponderado e modelos log-lineares. Resultados e conclusão: Houve boa concordância entre os métodos quanto à classificação na categoria 0 , ou seja, os dois métodos mostraram-se equivalentes para excluir o diagnóstico de silicose. Porém, para o diagnóstico da doença, caracterizado por classificação na categoria 1 ou superior, não foi obtida boa concordância entre os métodos. (J Pneumol 2001;27(4):199-205)
\end{abstract}

\section{Comparative study of high resolution computer-assisted tomography with chest radiograph in the diagnosis of silicosis incipient cases}

Introduction: At present, chest radiography $(C R)$ is the main instrument used in the diagnosis of silicosis, following the International Labor Organization (ILO) recommendations. In incipient cases, the interpretation of radiographs is difficult and disagreement may occur, even among experienced readers. Recently, the possibility of evaluating incipient cases by using high resolution computed tomography (HRCT) has been considered. Objective: To compare CR with $H R C T$ results. Patients and methods: An original group of 135 ex-miners were evaluated by $C R$, according to ILO recommendations, examined by three readers, from November 1997 to December 1999. HRCT was indicated to 68 patients whose median profusion readings was $1 / 0$ or below. HRCT results were examined by two readers and, in cases of disagreement, a third reader was consulted. HRCT results were classified according to micronodule profusion into categories 0

\footnotetext{
* Trabalho ganhador do III Prêmio Nacional de Pesquisa em Pneumologia em 2000. Resumo da Dissertação de Mestrado apresentada em 29/9/00 ao Programa de Pós-Graduação em Saúde Pública, Área de Concentração em Epidemiologia, da Universidade Federal de Minas Gerais.

1. Pneumologista do Ambulatório de Doenças Profissionais do Hospital das Clínicas da UFMG.

2. Professora Adjunta do Departamento de Estatística da UFMG.

3. Pneumologista/Pesquisador, Fundacentro-CTN.
}

\footnotetext{
4. Professor Titular de Radiologia do Departamento de Propedêutica Complementar da UFMG.

5. Professor Assistente do Instituto de Radiologia da FMUSP.

6. Radiologista do Centro de Imagem-MG.

7. Radiologista do Radiocentro-MG.

8. Titular do Departamento de Medicina Preventiva e Social da UFMG

Endereço para correspondência - Ana Paula Scalia Carneiro, Rua Curitiba, 2.115, apto. 701 - 30170-122 - Belo Horizonte, MG. Tels. (31) 3335-6472 e 3273-3911; E-mail: apscalia@zipmail.com.br
} 
to 3. $C R$ and HRCT results were compared using McNemar test, weighed Kappa coefficient and loglinear models. Results and conclusion: There was good agreement among the methods in category 0 , so it was concluded that both methods are similar in excluding the diagnosis of silicosis. However, in category 1 or greater (diagnosis of the disease) a good agreement among the methods was not observed.

Descritores - Silicose. Radiografia torácica. Tomografia computadorizada por raios X. Pneumoconiose.

Key words - Silicosis. Thoracic radiography. Tomography X-ray computed. Pneumoconiosis.

\section{INTRODUÇÃO}

A silicose representa um sério problema, uma vez que, apesar de ser potencialmente evitável, apresenta altos índices de incidência e prevalência, especialmente nos países menos desenvolvidos. O diagnóstico é baseado na radiografia de tórax, em conjunto com histórias clínica e ocupacional coerentes ${ }^{(1)}$. A OIT padronizou a técnica adequada para obtenção de radiografias de boa qualidade, assim como a codificação das alterações radiológicas das pneumoconioses, de maneira reprodutivel ${ }^{(2,3)}$. Porém, os casos iniciais de silicose, como as demais doenças pulmonares intersticiais crônicas em fases precoces, são de difícil avaliação, com alterações radiológicas discretas, confundindo-se com estruturas normais do parênquima pulmonar. Os diagnósticos nessa situação são os que causam maior desacordo, mesmo entre leitores experientes ${ }^{(4)}$. Nesse estágio, pequenos desvios da técnica radiológica ideal podem ocasionar distorções no diagnóstico e a situação torna-se mais problemática se o leitor for inexperiente $^{(5)}$. Portanto, devido a tais dificuldades, é esperado que a TCAR melhore a avaliação de imagens, assim como ocorre em várias outras doenças intersticiais( ${ }^{(6)}$.

\section{MATERIAL E MÉTODOS}

Trata-se de um estudo de delineamento transversal, para comparação de dois instrumentos de detecção. A amostra que permitisse comparar RX com TCAR foi dimensionada em 68 pacientes, com poder do teste de $90 \%{ }^{(7)}$. Eles estavam entre os primeiros 135 periciados, pertencentes a um grupo de aproximadamente 1.500 ex-mineiros que movem ações judiciais contra o ex-empregador, alegando ser portadores de silicose. A pesquisa foi aprovada pelo Comitê de Ética em Pesquisa do Hospital das Clínicas da Universidade Federal de Minas Gerais-UFMG.

Utilizaram-se os seguintes instrumentos para propedêutica:

a) Anamneses clínica e ocupacional padronizadas;
Siglas e abreviaturas utilizadas neste trabalho

CVF - Capacidade vital forçada

Kvp - Quilovoltagem

$\mathrm{mA}$ : Miliamperagem

OIT - Organização Internacional do Trabalho

RX - Radiografia

TBC - Tuberculose

TCAR - Tomografia computadorizada de alta resolução

$\mathrm{VEF}_{1}$ - Volume expiratório forçado no $1^{\circ}$ segundo

$\mathrm{UH}$ - Unidades Hounsfield

b) RX de tórax nos padrões da OIT, realizadas em uma única clínica radiológica, com leitura independente feita por três leitores experientes em pneumoconioses, sem o conhecimento de dados clínicos e/ou ocupacionais. A classificação foi feita de forma completa (escala semiquantitativa de 12 pontos, segundo padronização da OIT) e resumida $(0 /-, 0 / 0,0 / 1=0 ; 1 / 0,1 / 1,1 / 2=1 ; 2 / 1,2$ / $2,2 / 3=2 ; 3 / 2,3 / 3,3 /+=3$ ). Os resultados foram sumarizados pela mediana das leituras. Aceitaram-se apenas exames de qualidade 1 ou 2;

c) Testes de função pulmonar constituídos por espirometria, realizada de acordo com o Consenso Brasileiro de Espirometria ${ }^{(8)}$ e teste de caminhada de seis minutos;

d) TCAR indicadas nos casos com mediana das três leituras radiológicas menor ou igual a $1 / 0$, com a seguinte técnica: aparelho Siemens, modelo Somaton Plus-4; cortes de $1 \mathrm{~mm}$ de espessura; matriz de reconstituição de 512 x 512; tempo de duração de cada corte de um segundo; janela de nível -700 a $-800 \mathrm{UH}$; abertura de 1.000 a 1.600UH; Kvp de 140; mA de 240; algoritmo de reconstituição de alta resolução espacial; formatação em seis imagens por filme. Os cortes foram obtidos em posição de decúbito dorsal (em alguns casos utilizou-se ainda decúbito ventral), sem emprego de contraste venoso. Os exames foram avaliados por três radiologistas experientes, de diferentes serviços, não participantes da etapa das leituras radiológicas. Nos casos em que ocorreu divergência das leituras dos dois primeiros, foi realizada avaliação pelo terceiro radiologista. A graduação dos micronódulos (Quadro 1) seguiu a proposta de Begin et al..$^{(9)}$, classificando-os em quatro categorias, de acordo com sua profusão, de modo similar ao que se faz com a radiografia de tórax (classificação resumida da OIT). Os resultados foram 


\begin{tabular}{|c|c|}
\hline & \multicolumn{1}{c|}{$\begin{array}{c}\text { QUADRO 1 } \\
\text { Descrição das quatro categorias de graduação } \\
\text { dos micronódulos encontrados na TCAR }\end{array}$} \\
\hline Categoria & \multicolumn{1}{c|}{ Descrição } \\
\hline 0 & $\begin{array}{l}\text { Ausência de micronódulos } \\
1\end{array}$ \\
2 & $\begin{array}{l}\text { Micronódulos presentes, porém sem borramento } \\
\text { dos vasos } \\
\text { Micronódulos presentes, com algum borramento } \\
\text { dos vasos } \\
\text { Micronódulos presentes com acentuado borramen- } \\
\text { to dos vasos }\end{array}$ \\
\hline Adaptado de Begin et al.(9)
\end{tabular}

sumarizados pela mediana das leituras. O enfisema foi classificado de acordo com sua extensão, conforme proposta de Begin et al. ${ }^{(10)}$.

Foram realizadas análises descritiva e comparativa dos dados; na última aplicou-se o teste de McNemar, cálculos de coeficientes de concordância e ajuste de modelos loglineares ${ }^{(11)}$. Utilizaram-se os programas estatísticos EpiInfo, StatXact, SAS e Minitab.

\section{RESULTADOS}

\section{Análise descritiva}

As médias e os desvios padrões (entre parênteses) foram os seguintes: idade de 57,29 anos $(9,53)$, tempo de exposição à sílica de 15,25 anos $(5,98)$ e tempo de afastamento da exposição de 15,90 anos $(9,60)$. Em relação à espirometria (comparando-se com o previsto para brasileiros), a CVF foi de $98,10 \%(18,89)$ e o $\mathrm{VEF}_{1}$, de $94,22 \%$ $(24,21)$. Quanto à mediana das três leituras radiológicas, a distribuição dos 68 pacientes foi a seguinte: 21 na categoria 0/0; 34 na 0/1 e 13 na 1/0.

Os diagnósticos obtidos através da TCAR foram: enfisema - 19 casos, sendo o tipo centrolobular o mais freqüente (55\%); calcificação linfonodal - 19 casos, sendo um deles do tipo egg shell, 14 do tipo "outras" e quatro apresentavam os dois tipos; linfonodomegalia intratorácica - 18 casos; tuberculose - 12 casos de seqüelas típicas; espessamento de paredes brônquicas e/ou bronquiectasias - 14 casos; micronódulos compatíveis com silicose - dez casos, com identificação de confluências em dois deles; opacificação do tipo "vidro fosco" - sete casos, sendo que em cinco deles o padrão foi compativel com bronquiolite respiratória tabágica, um caso com alveolite alérgica extrínseca e um com proteinose alveolar; nódulo pulmonar solitário - três casos; imagem compatível com neoplasia pulmonar (massa) - dois casos.

\section{Análise comparativa}

Comparação de proporções dos resultados de RX e TCAR de tórax

Utilizou-se o teste de McNemar, que se presta para comparação de dois grupos pareados, com variável dicotômica, para comparar os diagnósticos de silicose (micronódulos), enfisema e seqüela de TBC verificados pelos dois métodos, RX e TCAR de tórax. Os achados foram classificados como ausentes ou presentes.

Em relação à silicose (Tabela 1), houve concordância dos resultados de RX e TCAR em 55 casos e divergência em 13, sendo que a TCAR acrescentou cinco novos casos de silicose em relação à RX e, por outro lado, afastou oito casos. Nota-se que não houve diferença estatisticamente significativa entre os dois métodos $(p=0,58)$.

No entanto, em relação ao diagnóstico de enfisema $e$ seqüela de TBC, houve significativo maior número de diagnósticos realizados pela TCAR do que pela RX, com valores p do teste, respectivamente, de 0,0013 e 0,0391.

\section{Análise de concordância com utilização do coefi- ciente Kappa}

A) Intramétodo: Antes de realizar a análise de concordância intermétodos, estudou-se o comportamento da concordância intramétodo, ou seja, entre os leitores de cada método. A avaliação foi feita através do coeficiente Kappa ponderado e, em alguns casos, foi necessário o procedimento de Monte Carlo para obtenção de resultados exatos ${ }^{(12)}$. Inicialmente, foram realizados cálculos utilizando-se os resultados com classificação nas 12 categorias radiológicas $e$, posteriormente, com a classificação resumida, a fim de tornar comparáveis os resultados da concordância entre leitores de RX com os de TCAR. Observa-se através dos resultados do coeficiente Kappa (Tabela 2) que não houve boa concordância, apesar de, em geral, terem sido obtidos bons percentuais de leituras concordantes, tanto entre os leitores de RX quanto entre os de TCAR.

B) Intermétodos (RX/TCAR de tórax): Observa-se na Tabela 1 que existe boa percentagem de leituras con-

TABELA 1

Distribuição do diagnóstico de silicose (micronódulos) através dos dois métodos: tomografia computadorizada de alta resolução (TCAR) e radiografia (RX) de tórax nos 68 pacientes estudados

\begin{tabular}{ccccc} 
& & \multicolumn{3}{c}{ TCAR } \\
\cline { 3 - 5 } & & Positivo & Negativo & Total \\
\hline \multirow{3}{*}{ RX } & Positivo & 5 & 8 & 13 \\
& Negativo & 5 & 50 & 55 \\
& Total & 10 & 58 & 68 \\
\hline
\end{tabular}


cordantes entre RX e TCAR (81\%), porém o valor do coeficiente Kappa ponderado o resultado foi de apenas 0,32 com intervalo de $95 \%$ do valor de confiança de 0,04; 0,61 . Sabe-se que esse coeficiente pode apresentar várias limitações, como a dependência em relação à prevalência da característica em estudo(13). Na casuística estudada, houve grande prevalência de classificações na categoria 0 e poucas na categoria 1 . Nota-se, por exemplo, que, em relação à mediana das classificações da TCAR, houve $85 \%$ na categoria 0 e $15 \%$ na categoria 1 . Portanto, quando, por exemplo, dois observadores classificam separadamente cada indivíduo da amostra em uma escala ordinal e baixo valor Kappa é obtido, torna-se necessária melhor compreensão das estruturas de concordância e discordância ${ }^{(14)}$. Para melhor analisar os resultados, existem algumas alternativas descritas na literatura, destacando-se o ajuste de modelos log-lineares.

\section{Análise da concordância através de modelos log- lineares}

O uso dos modelos log-lineares permite análise mais completa e informativa a respeito da concordância entre os avaliadores e entre os métodos. A Tabela 3 apresenta as freqüências das 14 configurações obtidas dos resultados dos leitores de RX e TCAR, aqui denominados como 1,2 e 3 para RX e 4 e 5 para TCAR. Observa-se que em 24 casos houve concordância dos cinco leitores na categoria 0 , o mesmo não acontecendo para a categoria 1 , isto é, não houve nenhum caso em que os cinco leitores classificassem como categoria 1 . A classificação na categoria 2 foi utilizada em apenas três casos.

Com a finalidade de avaliar a estrutura de concordância entre leitores e entre os métodos, foram considerados

TABELA 2

Estimativa e intervalo de confiança (IC) de $95 \%$ exatos para o coeficiente Kappa ponderado e percentual de leituras concordantes entre os leitores de radiografia (RX) e tomografia computadorizada de alta resolução (TCAR) de tórax

\begin{tabular}{cccc}
\hline \multirow{2}{*}{$\begin{array}{c}\text { Método } \\
\text { (n' de categorias) }\end{array}$} & \multicolumn{3}{c}{ Leitores } \\
\cline { 2 - 4 } & $\mathbf{1} \mathbf{e ~ 2}$ & $\mathbf{1} \mathbf{e ~ 3}$ & $\mathbf{2 ~ e ~ 3}$ \\
\hline \multirow{2}{*}{$\mathrm{RX}(12)$} & 0,45 & 0,26 & 0,26 \\
& $(0,32 ; 0,58)$ & $(0,09 ; 0,44)$ & $(0,13 ; 0,39)$ \\
& $49 \%$ & $43 \%$ & $35 \%$ \\
\hline RX (4) & 0,45 & 0,30 & 0,15 \\
& $(0,26 ; 0,65)$ & $(0,06 ; 0,53)$ & $(0,003 ; 0,30)$ \\
& $72 \%$ & $79 \%$ & $69 \%$ \\
\hline TCAR (4) & 0,14 & 0,16 & 0,33 \\
& $(-0,02 ; 0,31)$ & $(-0,20 ; 0,51)$ & $(0,13 ; 0,53)$ \\
& $68 \%$ & $85 \%$ & $60 \%$ \\
\hline
\end{tabular}

os seguintes modelos: 1) Independência: assume não existir nenhuma estrutura de concordância; 2) Concordância global entre leitores: assume que há concordância se todos os leitores classificam na mesma categoria; 3) Quaseconcordância entre leitores: assume que há concordância se pelo menos quatro leitores classificam na mesma categoria, independente do método e da categoria de classificação; 4) Concordância global entre métodos: assume que há concordância se dois leitores de RX concordam com os dois leitores de TCAR, independente da categoria; 5) Concordância entre métodos com categorias heterogêneas: assume que há concordância se dois leitores de $\mathrm{RX}$ concordam com dois leitores de TCAR, levando em consideração as categorias; 6) Concordância entre pares de leitores com categorias heterogêneas: assume que há concordância se dois leitores de RX concordam com dois leitores de TCAR, levando em consideração as categorias e os pares que concordam no método de RX.

A partir dos dados da Tabela 3, foram ajustados modelos correspondentes às estruturas 1 a 6 . A comparação dos modelos foi feita através do teste da razão da verossimilhança, que é baseada na diferença dos deviances e dos graus de liberdade dos modelos comparados. Quanto menor o valor obtido, melhor o modelo se ajustou à sua definição. O fluxograma dos modelos hierárquicos é apresentado na Figura 1.

Através dos modelos log-lineares constatou-se que existe uma estrutura de concordância entre os leitores de RX com os de TCAR, que foi melhor descrita pelo modelo 5 , que considera existência de concordância se dois leitores de RX concordam com dois leitores de TCAR, levando em

TABELA 3

Freqüência das combinações de classificações por radiografia e tomografia computadorizada de alta resolução (TCAR) de tórax

\begin{tabular}{cccccc}
\hline \multicolumn{4}{c}{ Leitores } & & \multicolumn{2}{c}{ Freqüências } \\
\cline { 2 - 5 } \multicolumn{2}{c}{ Radiografia } & \multicolumn{2}{c}{ TCAR } & \\
\cline { 2 - 5 } $\mathbf{1}$ & $\mathbf{2}$ & $\mathbf{3}$ & $\mathbf{4}$ & $\mathbf{5}$ & \\
\hline 0 & 0 & 0 & 0 & 0 & 24 \\
0 & 0 & 0 & 0 & 1 & 13 \\
0 & 0 & 0 & 1 & 1 & 1 \\
1 & 0 & 0 & 0 & 0 & 6 \\
0 & 1 & 0 & 0 & 0 & 9 \\
0 & 1 & 0 & 0 & 1 & 2 \\
1 & 1 & 0 & 0 & 0 & 3 \\
1 & 1 & 0 & 0 & 1 & 1 \\
1 & 1 & 0 & 1 & 0 & 2 \\
1 & 1 & 0 & 1 & 1 & 3 \\
1 & 1 & 1 & 0 & 1 & 1 \\
1 & 1 & 1 & 0 & 2 & 1 \\
1 & 2 & 0 & 0 & 1 & 1 \\
1 & 2 & 0 & 1 & 1 & \\
\hline
\end{tabular}




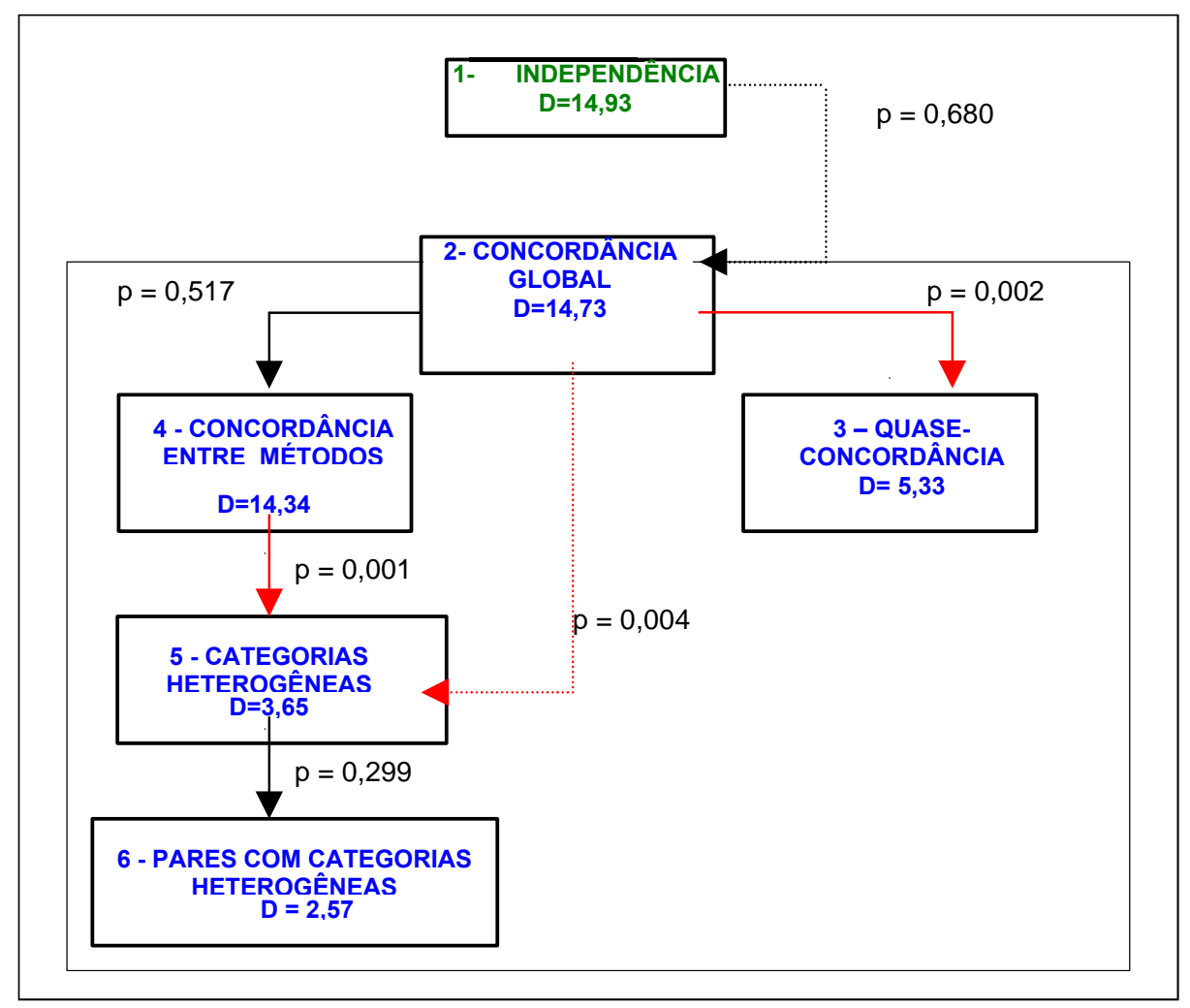

Figura 1 - Fluxograma dos modelos hierárquicos com valores p usados na comparação entre os mesmos e valores dos respectivos deviances $(D)$

consideração a categoria de classificação, isto é, distinguindo as concordâncias na categoria 0 das concordâncias na categoria 1 .

\section{DISCUSSÃO}

Embora já existam estudos descritivos dos achados de silicose através da $\operatorname{TCAR}^{(6,15)}$, a literatura ainda é carente de análises comparativas desta com achados anatomopatológicos em grupos expostos à sílica, através das quais seja possível calcular sensibilidade e especificidade desse método, como já realizado em outras doenças pulmonares intersticiais ${ }^{(16,17)}$. Através de revisão da literatura localizou-se apenas um estudo no qual foi feita essa comparação(18), podendo ser calculadas, através dos dados apresentados, a sensibilidade da TCAR e a da RX, para a categoria $0 / 1$, obtendo-se, respectivamente, valores de $86 \%$ e de $57 \%$.

A possibilidade de a TCAR de tórax ser utilizada para diagnosticar com maior precisão lesões mínimas de silicose têm sido avaliada através da comparação dos achados da TCAR com os de RX, testes de função pulmonar, variáveis clínicas e ocupacionais, através dos quais pode avaliar-se indiretamente o desempenho dos métodos de imagem, porém, ainda sem conclusões consensuais.
Alguns estudos demonstraram, através da TCAR, melhor explicação para a disfunção pulmonar apresentada pelos pacientes, por intermédio da identificação de áreas de enfisema pulmonar não percebidas por RX e/ou por complicações decorrentes da própria silicose, como, por exemplo, coalescência de lesões, mesmo com utilização de TC convencional ${ }^{(19-21)}$.

Em 1991, Begin et al. ${ }^{(21)}$ submeteram à TCAR e TC convencional expostos à sílica com RX classificadas como 1 ou 0 . A TC e TCAR acrescentaram $12 \%$ de novos diagnósticos de confluência, além de $40 \%$ de portadores de micronódulos não identificados à $\mathrm{RX}$ de tórax. Em relação ao grupo que tinha RX alterada, a TC e TCAR não confirmaram a presença de micronódulos em $14 \%$ deles. Em 1993, Cowie et al. ${ }^{(22)}$ avaliaram 70 expostos à sílica através de TCAR, que acrescentou apenas dois diagnósticos de silicose em relação à RX e afastou nove casos. Em 1995, Talini et al. ${ }^{(23)}$ estudaram 27 expostos à sílica, comparando os resultados de RX com TCAR e testes de função pulmonar. A TCAR acrescentou seis novos diagnósticos de silicose e afastou cinco, previamente identificados pela RX de tórax. A discriminação das categorias de profusão de micronódulos, feita através da TCAR, mostrou melhores associações com os testes de função pulmonar. Em 1998, Gevenois et al. ${ }^{(24)}$ avaliaram um grupo de 35 ex- 
postos à sílica e 48 expostos ao carvão, que foram submetidos a RX, TCAR de tórax e testes de função pulmonar. A RX identificou como portadores de pneumoconioses 27 pacientes e a TCAR, 46, diferença essa que foi estatisticamente significativa. A diferenciação dos grupos através da TCAR não mostrou associação com os resultados de função pulmonar.

Quanto à avaliação de concordância entre leitores, existem vários estudos, especialmente em relação a radiografias ${ }^{(13,25,26)}$. Percebe-se tendência de piores índices de concordância nos casos iniciais e nos filmes de pior qualidade, e melhores índices nas casuísticas compostas por RX classificadas nas diversas categorias, incluindo as mais avançadas $^{(9,26,27)}$. Em nosso estudo, somente foram incluídos casos incipientes, o que pode ter contribuído para os baixos coeficientes de concordância encontrados.

Em relação à TCAR, a inexistência de modelos, através dos quais se faria a classificação visual comparativa, de modo semelhante ao que se faz com a RX, pode ser uma desvantagem. Embora existam propostas da padronização da classificação tomográfica, especialmente em relação às alterações secundárias ao asbesto, ainda não existe aceitação geral da mesma ${ }^{(28)}$. Uma proposta oferece, inclusive, auxílio de programas de computação(29). Apesar disso, segundo a literatura, alguns autores encontraram melhor concordância entre leitores de TCAR do que de $\mathrm{RX}$ de tórax ${ }^{(9,23)}$, o que não foi verificado em nosso estudo.

A realização de leituras múltiplas, tanto de RX como de TCAR, é fundamental na comparação dos dois métodos, uma vez que há variabilidade interindividual nas leituras, conforme observado em nosso estudo, através dos diferentes resultados encontrados ao comparar diversos pares de leitores. Entretanto, o coeficiente Kappa, apesar de amplamente utilizado na literatura, não é adequado para análise de tabelas "desbalanceadas", como a dos dados em questão, por ser influenciado pela prevalência da característica em estudo, assim como pelo número de categorias utilizadas na classificação.

Na literatura, um trabalho recente $e^{(30)}$ comparou os dois métodos, RX e TCAR, através do coeficiente Kappa, conforme realizado em nosso estudo. Trata-se de uma casuística de 75 expostos à sílica e poeiras mistas, nas diversas fases da doença. O resultado do Kappa foi de 0,52. Em nosso estudo, encontrou-se pior concordância, com resultado de 0,32 , o que pode ser parcialmente justificado por tratar-se apenas de casos incipientes, de avaliação mais difícil.

Além da importância do registro de leituras múltiplas, deve-se considerar que o método tradicional que resume as leituras em uma única medida de tendência central, como a mediana, pode causar perda de informação. Esta dificuldade estimula o uso de métodos de análise apro- priados (por exemplo, modelos log-lineares). Em nosso estudo, foi demonstrado, através dos modelos log-lineares, existir boa concordância entre RX e TCAR na classificação em categoria 0 , ou seja, para excluir o diagnóstico de silicose, os dois exames são semelhantes, desde que realizados e interpretados nos padrões estabelecidos nesta pesquisa. Não foi demonstrada boa concordância na classificação em categoria 1, o que, na ausência de padrão ouro, não nos permite afirmar a superioridade da TCAR em relação à radiografia. Existem dificuldades nos dois métodos na identificação de micronódulos em pequena profusão. Na radiografia existe a limitação imposta pela sobreposição de imagens e na TCAR há dificuldade na diferenciação entre micronódulos em baixa profusão e vasos pulmonares, que, em cortes transversais, são estruturas semelhantes.

Recentemente, tem sido avaliado o uso da técnica de alta resolução com aquisição volumétrica e projeção de máxima intensidade, para melhor distinção de micronódulos e vasos nas pneumopatias intersticiais difusas ${ }^{(31,32)}$, que parece ser promissor.

Em relação ao enfisema, a literatura mostra que a TCAR é um método diagnóstico muito mais sensivel que a RX de tórax ${ }^{(33,34)}$, podendo diagnosticar casos mesmo em pacientes com espirometria e radiografia de tórax normais( ${ }^{(35)}$. No nosso estudo, em relação ao enfisema e à TBC, a TCAR mostrou maior número de casos do que a radiografia, o que pode ter grande importância nos diagnósticos diferenciais da silicose, assim como contribuição na melhor avaliação dos sintomas e da incapacidade.

\section{CONCLUSÕES}

Há necessidade de aplicação de protocolos com padronização de técnica, conceitos e classificações de lesões nos serviços que já utilizam a TCAR em expostos à silica.

A criação de modelos visuais de tomografias em expostos à sílica, de modo semelhante ao que já existe para radiografias, poderá diminuir a variabilidade interindividual nas leituras.

Há necessidade de mais estudos para esclarecer o papel da TCAR no diagnóstico precoce da silicose, com identificação de critérios para sua realização, visando melhor aproveitamento do exame, através da criação de algoritmos ou fluxogramas que avaliem inclusive a questão do custo-benefício.

\section{REFERÊNCIAS}

1. Algranti E, Capitani EM, Bagatin E. Sistema respiratório. In: Mendes R. Patologia do trabalho. 2a ed. Rio de Janeiro: Atheneu, 1995;99137. 
2. International Labour Office. Guidelines for use of ILO International Classification of Radiographs of Pneumoconioses. Geneva, 1980. (Occupational Safety and Health Series n. 22).

3. Organização Internacional do Trabalho. Fundacentro. Leitura radiológica das pneumoconioses. São Paulo: Fundacentro, 1994;72 p.

4. Graham WGB. Quartz and silicosis. In: Banks DE, Parker JE. Occupational lung diseases: an international perspective. 1st ed. London: Chapman and Hall Medical, 1998;191-212.

5. Algranti E. Métodos de investigação em doenças ocupacionais pulmonares. J Pneumol 1994;20:165-173.

6. Remy-Jardin M, Remy J, Farre I, Marquette CH. Computed tomographic evaluation of silicosis and Coal Workers Pneumoconiosis. Radiol Clin North Am 1992;30:1155-1176.

7. Lu Y, Beam JA. On the sample size for one-sided equivalence of sensitivities based upon McNemar test. Stat Med 1995;14:1831-1839.

8. Sociedade Brasileira de Pneumologia e Tisiologia. I Consenso Brasileiro de Espirometria. J Pneumol 1996;22.

9. Begin R, Bergeron D, Samson L, Boctor M, Cantin A. CT assessment of silicosis in exposed workers. AJR Am J Roentgenol 1987;148:509514.

10. Begin R, Filion R, Ostiguy G. Emphysema in silica and asbestos exposed workers seeking compensation. Chest 1995;108:647-655.

11. Silva EF. Variabilidade observacional em epidemiologia: uma metodologia de avaliação das estruturas de concordância com dados ordinais categorizados em delineamentos com painéis fixos. Brasília: Universidade de Brasília, Faculdade de Ciências da Saúde, 1998. 147p. [Tese, Doutorado em Ciências da Saúde].

12. Fishman GS. Monte Carlo: concepts, algorithms and applications. New York: Springer, 1996;698p.

13. Muir DCF, Bernholz CD, Morgan WKC, Roos JO, Chan J, Maehle W, et al. Classification of chest radiographs for pneumoconiosis: a comparison of two methods of reading. Br J Ind Med 1992;49:869-871.

14. Silva EF, Perreira MG. Avaliação das estruturas de concordância e discordância nos estudos de confiabilidade. Rev Saúde Pública 1998;32: 383-393.

15. Remy-Jardim M, Remy J, Deffontaines C, Duhamel A. Assessment of diffuse infiltrative lung disease: comparison of conventional CT and high-resolution CT. Radiology 1991;181:157-162.

16. Padley SPG, Hansell DM, Flower CDR, Jennings P. Comparative accuracy of high resolution computed tomography and chest radiography in the diagnosis of chronic diffuse infiltrative lung disease. Clin Radiol 1991;44:222-226.

17. Padley SPG, Adler B, Müller N. High resolution computed tomography of the chest: current indications. J Thorac Imaging 1993;8:189199.

18. Olivetti L, Grazioli L, Milanesio L, Provezza A, Chiodera P, Tassi G, et al. Definizione anatomo-radiologica della silicosi minima interstiziale e contributo diagnostico della tomografia computerizzata con alta risoluzione. Radiol Med 1993;85:600-605.

19. Bergin CJ, Müller NL, Vedal S, Chan-Yeung M. CT in silicosis: correlation with plain films and pulmonary function tests. AJR Am J Roentgenol 1986;146:477-483.
20. Begin R, Ostiguy G, Cantin A, Bergeron D. Lung function in silicaexposed workers: a relationship to disease severity assessed by CT scan. Chest 1988;94:539-545.

21. Begin R, Ostiguy G, Fillion R, Colman N. Computed tomography scan in early detection of silicosis. Am Rev Respir Dis 1991;144:697-705.

22. Cowie RL, Hay M, Thomas RG. Association of silicosis, lung dysfunction and emphysema in gold miners. Thorax 1993;48:746-749.

23. Talini D, Paggiaro PL, Falaschi F, Battolla L, Carrara M, Petrozzino M, et al. Chest radiography and high resolution $\mathrm{CT}$ in the evaluation of workers exposed to silica dust: relation with functional findings. Occup Environ Med 1995;52:262-267.

24. Gevenois PA, Sergent G, Maertelaer V, Gouat J, Yernault JC, Vuyst P. Micronodules and emphysema in coal mine or silica exposure: relation with lung function. Eur Respir J 1998;12:1020-1024

25. Musch DC, Landis R, Higgins ITT. An application of Kappa-type analyses to interobserver variation in classifying chest radiographs for pneumoconiosis. Stat Med 1984;3:73-83.

26. Welch LS, Hunting KL, Balmes L, Bresnitz EA, Guidotti TL, Lockey JE, et al. Variability in the classification of radiographs using the 1980 International Labor Organization classification for pneumoconiosis. Chest 1998;114:1740-1748.

27. Kreiss K, Zhen B. Risk of silicosis in a Colorado mining community. Am J Ind Med 1996;30:529-539.

28. Kraus T, Raithel HJ, Hering KG. Evaluation and classification of highresolution computed tomography findings in patients with pneumoconiosis. Int Arch Occup Environ Health 1996;68:249-254.

29. Kraus T, Raithel HJ, Ilehnert G. Computer-assisted classification system for chest X-ray and computed tomography findings in occupational lung disease. Int Arch Occup Environ Health 1997;69:482-486.

30. Biscaldi G, Fonte R, Paita L, Vittadini G, Caprotti M. La tomografia computerizzata ad alta risoluzione nella diagnosi di silicosi e di pneumoconiosi da rischio misto. G Ital Med Lav Ergon 1999;21:278-286.

31. Remy-Jardin M, Remy J, Artaud D, Deschildre F, Duhamel A. Diffuse infiltrative lung disease: clinical value of sliding-thin-slab maximal intensity projection $\mathrm{CT}$ scans in the detection of mild micronodular patterns. Radiology 1996;200:333-339.

32. Vernhet H, Bousquet C, Vergnes C, Thiebault C, Lesnik A, Durand G, et al. Apport de la TDM avec haute résolution volumique (TDM HRV) dans l'exploration des pneumopathies infiltratives diffuses. Rev Mal Respir 1999;16:188-197

33. Snider GL, Faling LJ, Rennard SI. Chronic bronchitis and emphysema. In: Murray JF, Nadel JA. Respiratory medicine. 2nd ed. Philadelphia: Saunders, 1994;1331-1397.

34. Fraser RS, Müller NL, Colman N, Paré PD. Chronic obstructive pulmonary disease. In: Diagnosis of diseases of the chest. 4th ed. Philadelphia: WB Saunders, 1999;2168-2263.

35. Klein JS, Gamsu G, Webb WR, Golden JA, Müller NL. High-resolution CT diagnosis of emphysema in symptomatic patients with normal chest radiographs and isolated low diffusing capacity. Radiology 1992;182: 817-821 